\title{
Inguinal Orchiectomy
}

National Cancer Institute

\section{Source}

National Cancer Institute. Inguinal Orchiectomy. NCI Thesaurus. Code C15724.

Surgical removal of a testis through an inguinal approach. 